\title{
An Efficient Design of Blood Vessel Image Extraction Using LBP Technique
}

\author{
G. Somasekhar ${ }^{1}$, Dr. O.Obulesu ${ }^{2}$, Dr. P. Appala naidu ${ }^{3}$, Dr.Rokesh Kumar Yarava ${ }^{4}$ \\ \{giddalurisomasekhar@gmail.com ${ }^{1}$, obulesh194@gnits.ac.in², appalanaidu.p@raghuenggcollege.in ${ }^{3}$, \\ rokeshy1@gmail.com ${ }^{4}$ \} \\ Associate Professor, Department of Computer Science and Engineering, CMR Technical Campus, \\ Hyderabad, Telangana-501401 ${ }^{1}$, Associate Professor, Department of IT, G.Narayanamma Institute of \\ Technology \& Science (Autonomous), Hyderabad, Telangana-500104², Associate Professor of CSE, \\ Raghu Engineering College, Dakamarri (V), Bheemunipatnam (M), Visakhapatnam (Dt), A.P.-531162. ${ }^{3}$ \\ Yarava, Associate Professor, Department of CSE, Chalapathi Institute of Engineering and \\ Technology(A), Guntur,A.P.-522006. ${ }^{4}$
}

\begin{abstract}
An efficient architecture of blood vessel image extraction is implemented. This system provides both physiological and behavioral characteristics which give the overview of retina, iris recognition, and face detection. The main intent of this project is to detect and measure the blood vessels present in the retina. This is done by using the classifier technique. By using classifier technique, the accuracy of the system will be increased. Image segmentation is applied to divide the number of cells available in the retina. The LBP technique will extract the features of input retinal images. Image feature extraction using LBP will extract the images which are featured. Imaging Library provides the interpreter with image editing capabilities. Hence this blood vessel image extractiongives effective results and this can be observed from simulation results.
\end{abstract}

Keywords: Blood Vessel Image Extraction. Classifier Technique, Image Segmentations, Histogram Equalization, Local Binary Pattern (LBP)..

\section{Introduction}

Eyes are the most significant organs in our body, called as organs of vision. Vein extraction is significant and the same number of eye sicknesses is perceived by assessing the vein. These veins convey crisp oxygenated blood from heart to sustain sustenances to tissues and cells present in retina and afterward convey the deoxygenated blood from eye to heart. There are for the most, two sorts of veins supply routes.

i) The veins that convey new blood.

ii) The veins that convey oxygenated blood

Photoreceptors move light into neural sign that are passed to the cerebrum through the optic nerves. A fundus camera system is normally utilized for capturing retinal images to save the retina condition. Retinal image contains important diagnostic data which helps to identify whether the retina is diseased or non-diseased. Blood vessels appear as networks of mesh-like structure or tree-like structure of either dark red or orange-red fibers that start inside the optic disc and dynamically which reduces the width. The morphological characteristics of the retina 
such as length, width, and branching is essential for diagnosis, screening, early detection and treatment of various diseases such as stroke, vein occlusions, glaucoma, hypertension, diabetes and arteriosclerosis which aid a appropriate detection and treatment in an early stage.

In addition, the investigation of retinal blood vessels can support in the assessment of retinal image segmentation in terms of the relationship between vessel and hypertensive retinopathy, retinopathy of prematurity, reflectivity, arteriolar narrowing, mosaic synthesis, biometric identification, foveola vascular region detection and computer-assisted laser surgery.

While cardiovascular and ophthalmologic diseases have a severe impact on human's life, so retinal blood vessels analysis is more significant. The patients might not notice a vision loss until it turned out to be serious; consequently, early diagnosis and appropriate treatment are necessary to prevent visual hinder and blindness. It has great significance in clinical applications to expose important information about retina for treatment. So it is mandatory to develop an efficient technique for segmentation of retinal vessel for diagnosis.

The process of dividing image into various regions is called as image segmentation, every region have different properties such as texture or colour. The pixels are arranged as background or foreground by using segmentation in the experiment of microarray. For every spotted DNA sequence, the fluorescence intensities can be determined.

Spot mask is produced by segmentation method, it contains foreground pixels set. In the foreground spots fluorescence signals mean intensity determination is known as spot intensity extraction [1]. Mean intensities are extracted which are matched to gene expression levels then transferred into biological conclusions by using data mining techniques. The problems of segmentation of microarray image are discussed in segmentation of microarray image.

The goal of segmentation is division of microarray image pixels into various groups or regions. Set of foreground pixels are in one group and background pixels are in another group. Here different types of pixels are existing like noisy pixels; these are contaminated pixels which are generated during microarray production and scanning. In the process of segmentation these pixels are removed from foreground region or background region [2].

Based on methods for classification of pixels, another type of pixels contains foreground region surrounded by edge pixels. Due to these pixels intensities presents in between background and foreground, adding or removing those causes SNRs are present. Usually two clusters are assumed that they are for the foreground pixels and $\mathrm{Co}_{2}$ for the background pixels. In this d-dimensional feature vector real values are used to show the features that are extracted from pixel $\mathrm{p}$.

The problem of segmentation contains assigning of every pixel of I, G or R to predefined classes. In segmentation method the output will be binary image B or white or black. In the image each pixel assigned by label, by using various statistical measures intensities of background and foreground can be manipulated. To find the process of typical objects from background which is called image segmentation. In vision systems first step is the image segmentation, for additional processing like recognition or description it is the basic step. From the images, important features are extracted by the segmentation. 
The image segmentation can also be stated that each pixel assigned to compositions of image. There are five types of image segmentation methods; they are hybrid methods, boundary detection methods, shape-based methods, threshold based or clustering methods and region growing methods. To get good results some application domains uses the different image segmentation methods such as motion image segmentation, color image segmentation, real time image segmentation and 3-D image segmentation. Most basic image segmentation method is the threshold or clustering technique. In these techniques,pixels are classified into different regions by getting the information about pixels and its neighbors [3].

Contour detection is focused by edge-based methods or Boundary detection. The segmentation of image depends on linking and edge finding or spatial discontinuity. Edge detection operators are Laplacian, Prewitt and Sobel operators. The image segmentation performed by method of region growing and depends on pixels spatial similarity [4].

Same intensity levels of neighboring pixels are grouped and by using this images are divided into connected regions. After that, merge the adjacent regions which contain sharpness or homogeneity of region boundaries. The shape of object which has to be segmented is used by shape based methods such as template matching and mathematical morphology. For image segmentation, there are different methods present. For analysis, microarray image special techniques are implemented. In microarray image, the characteristics are considered by these techniques [5].

\section{Related Work}

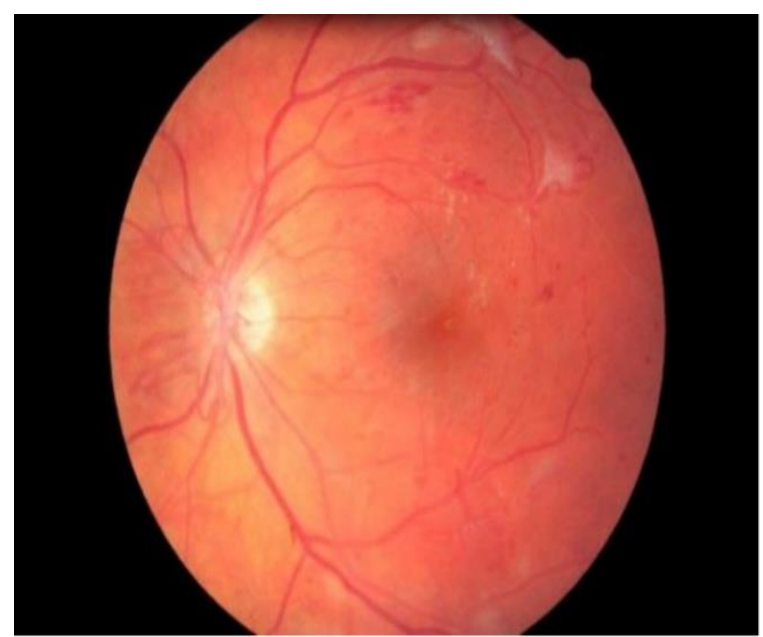

Fig. 1:STRUCTURE OF RETINA VESSEL

Segmentation can improve screening for retinopathy by decreasing the quantity of falsepositive results from the patient which is taken under various circumstances by depicting the optic disc region and fovea. In any case, manual detection of blood vessels is complicated since the blood vessels in a retinal image are obscure and low contrast. In modern healthcare community, medical imaging has turned into the most vital tool due to visual documentation 
and to record the patient's information for diagnosing. By employing computational approaches, for this reason would help in the efficient retinal analysis.

Image processing techniques are widely used in medical diagnosis for efficient segmentation. Pre-processing, feature extraction, segmentation, and classification are the common image processing techniques used for segmentation of retinal blood vessel. Automatic segmentation of retinal blood vessels is a prevailing tool for medical diagnostics and enhances the diagnostic performance of less specialized physicians. For this reason, the segmentation method is utilized because of accurate and reliable. The important process of segmentation is to distinguish features and the background from a given image. The basic strategies for retinal blood segmentation are supervised methods and unsupervised methods.

Supervised methods acquire segmentation results with labeled images whereas unsupervised methods do not need a labeled image. Automatic blood vessel segmentation in the images can help speed diagnosis and improve the diagnostic performance of less specialized physicians. A crucial step in feature extraction is the segmentation of the original image. This work focuses on developing a new approach for retinal blood vessel segmentation, to overcome the flaws of existing algorithms by comparing their performances to achieve the efficient performance. In this blood vessel image extraction, unsupervised method for segmentation of retinal blood vessel from color fundus retina image for classifying the image as disease affected blood vessel or non disease affected blood vessel. This proposed method is based on generating a feature vector for every image pixel by utilizing training samples (known classes) to design a classifier to classify these training samples into their subsequent classes and to classify the disease as diabetic retinopathy.

Here it is used to implement the structure. The main uses of the retina blood image extraction in image processing are given below:

1. It is used to read an image and save it in another image file format

2. It is used to read an color image and convert into gray scale image.

3. It is used to increase the contrast of image.

4. It is used to increase the brightness of image.

5. It is used to display the image name of images stored in directory.

\section{Proposed Method}

The below figure (2) shows the architecture of retina blood image extraction vessel using LBP. Here first an RGB image is taken. After that pre processing method is applied to that input image. By using histogram equalization method, the contrast of the obtained image is improved. Here classifier technique is implemented which uses both KNN (K-Nearest Neighbor) and RF (Random Forest) classifiers. Now after applying the classification technique image is segmented into number of pixels. After segmentation the features are extracted from that image. Hence from this one can observe the output image. 


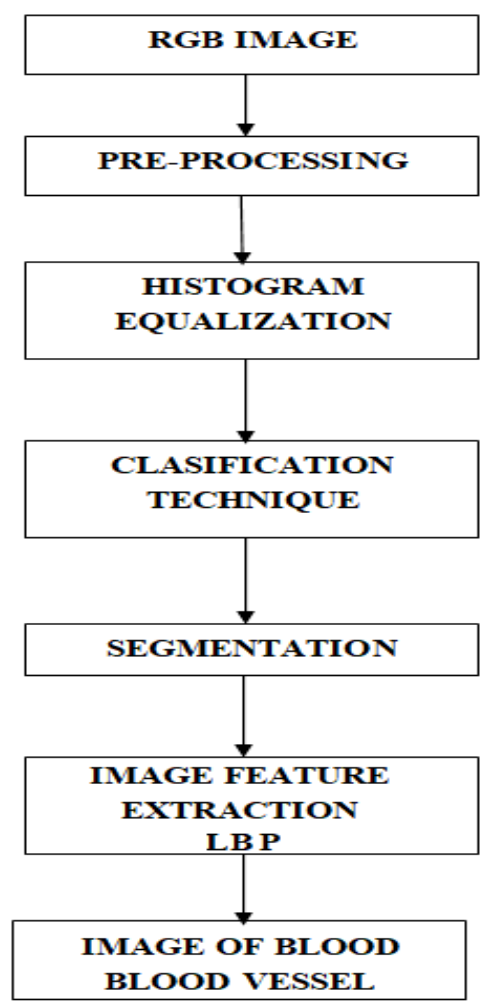

Fig. 2: RETINA BLOOD IMAGE EXTRACTION VESSEL

Pre processing step is improved to distinguish the suspicious district by the utilizing of the Pre-handling methods. The initial step of pre-handling is following the highlights which includes those exercises that are typically vital preceding real data audit and detail extraction, and are every now and again gathered as radiometric or geometric enhancements. For instance, film antiques or names, age and characteristics of the patient. Through the following calculation, film curios are dispensed. Now, the power estimation of the pixels are beginning from the essential line and the underlying segment is examined and the limit estimation of the film ancient rarities is found. The edge worth ought to be noted to surpass the edge esteems expelling from Blood Vessel Imagewipe out of the film ancient rarities high power esteem. Sooner or later in taking out film ancient rarities, the picture incorporates salt and pepper clamor.

Visual review of a great deal of pictures uncovers that the objects of interests have a dim worth that is not quite the same as the foundation. In these cases, the histogram will frequently demonstrate a bimodal dissemination. Thresholding is the least complex strategy for picture division. From a grayscale picture, thresholding can be utilized to make parallel pictures.

Morphological picture preparing is a gathering of non-straight tasks identified with the shape or morphology of highlights in a picture, for example, limits, skeletons, and so forth. In some random system, testing a picture with a little shape or format is called as an organizing component, which characterizes the district of intrigue or neighborhood around a pixel. Morphological administrators enlarge, disintegrate, open, and close can be connected through 
picture sifting to develop or psychologist picture areas, just as to expel or fill-in picture locale limit pixels. As zones of closer view pixels develop in size, gaps inside those locales become littler.

The yields for proposed technique comprises of different sorts of exactnesses, those are resemble RBF precision, Linear precision, Polygonal precision and Quadratic precision. A Radial Basis Function is a genuine esteemed capacity whose worth depends just on the good ways from the beginning, then again on the good ways from some other point, called as inside, so that, the RBF execution is prepared enough to have the RBF abused in various designing applications.

Precision and repeatability are two significant determinations of a direct movement stage. In spite of the fact that they are connected, precision and repeatability in straight movement frameworks are not something very similar. The engine, drive and actuator itself all impact these details. Now and then, repeatability could really compare to generally exactness. The overall significance of the two relies with an intensive comprehension of your application.

Pre-preparing procedures is utilized to improve the identification of the suspicious district from blood vessel image. The pre-handling and upgrade techniques comprises of two stages; first the evacuation of film antiquities, for example, names and X-beam imprints are expelled from the Blood Vessel Image utilizing following calculation. Second, the evacuation of high recurrence parts utilized Ant Colony Optimization (ACO) system.

\section{Results}

The below figure (3) shows the input fundus image.

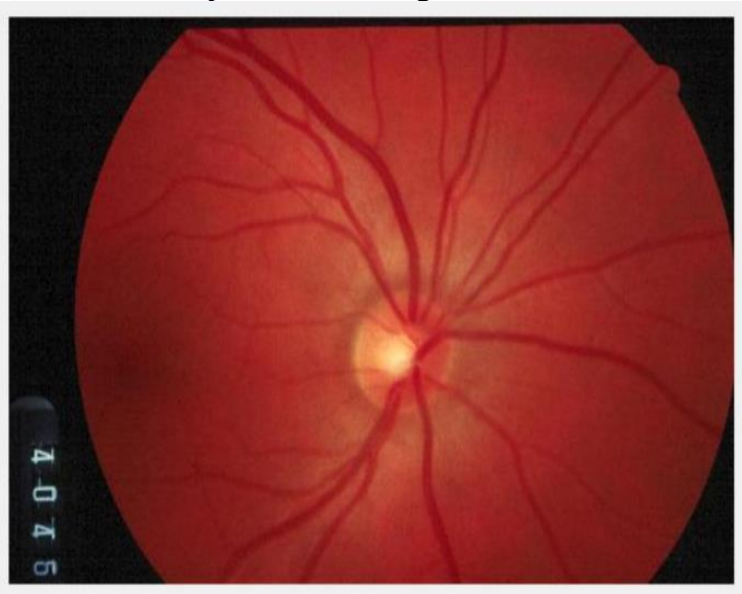

Fig. 3: INPUT FUNDUS IMAGE

The below figure (4) shows the gray scale image. The input image is converted into gray scale image. 


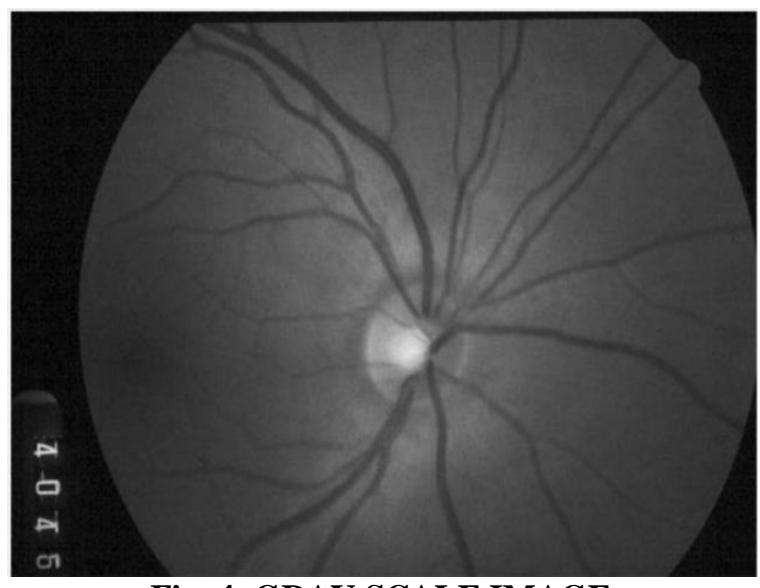

Fig. 4: GRAY SCALE IMAGE

The below figure (5) shows the pre processing image. The converted image is pre processed.

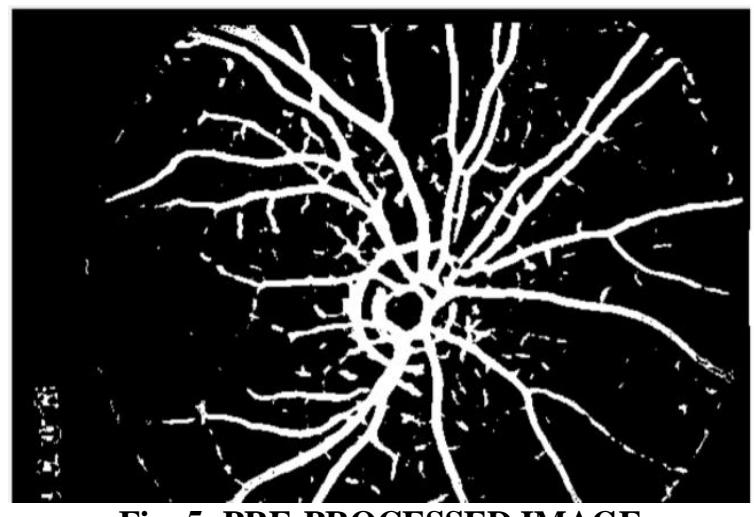

Fig. 5: PRE-PROCESSED IMAGE

The below figure (6) shows the output KNN classifier image. The processed image is classified using KNN.

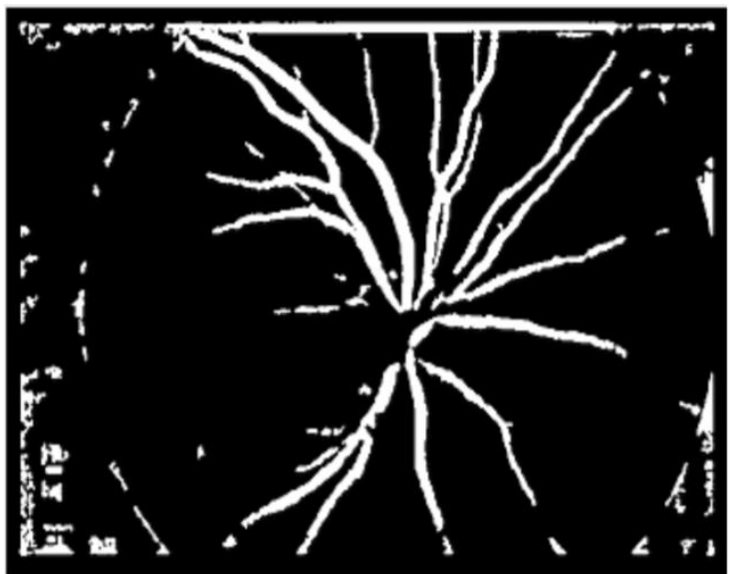

Fig. 6: OUTPUT OF KNN CLASSIFIER 
The below figure (7) shows the extracted blood vessel image.

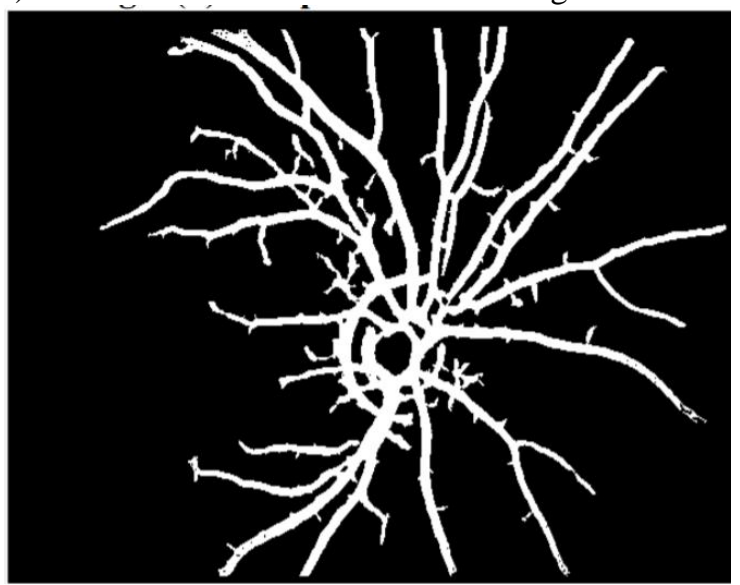

Fig. 7: EXTRACTED BLOOD VESSEL

Table. 1: ANALYSIS OF PROPOSED AND EXISTING SYSTEM

\begin{tabular}{|c|c|c|c|}
\hline S.No & $\begin{array}{l}\text { Name of the } \\
\text { parameter }\end{array}$ & $\begin{array}{l}\text { Blood Vessel } \\
\text { Image } \\
\text { Extraction }\end{array}$ & $\begin{array}{l}\text { Blood Vessel } \\
\text { Image } \\
\text { Extraction } \\
\text { using LBP } \\
\text { technique }\end{array}$ \\
\hline 1 & Accuracy & $31 \%$ & $95 \%$ \\
\hline 2 & $\begin{array}{l}\text { Number of } \\
\text { Hidden } \\
\text { Nodes }\end{array}$ & 52 & 6 \\
\hline 3 & $\begin{array}{l}\text { Learning } \\
\text { Rate }\end{array}$ & 0.08 & 0.05 \\
\hline 4 & $\begin{array}{l}\text { Number of } \\
\text { Maximum } \\
\text { Iterations }\end{array}$ & 500 & 1000 \\
\hline
\end{tabular}

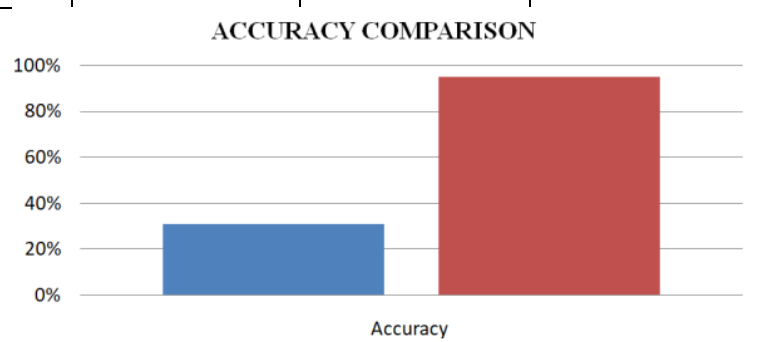

n Blood Vessel Image Extraction

n Blood Vessel Image Extraction using LBP technique

Fig. 8: ACCURACY COMPARISON 
The above figure (8) shows the accuracy comparison of existing and proposed system. Compared to existing, proposed system increases the efficiency.

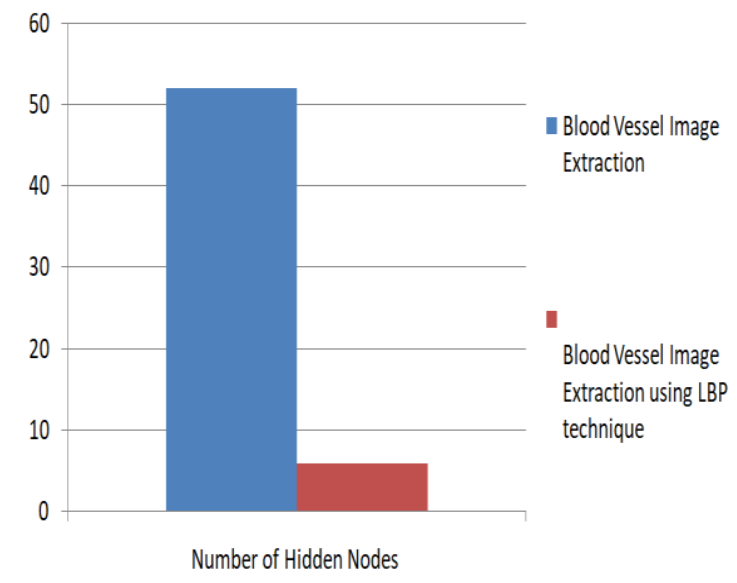

Fig. 9: COMPARISON OF NUMBER OF HIDDEN NODES

The above figure (8) shows the comparison of number of hidden nodes in existing and proposed system.

\section{Conclusion}

Hence the blood vessel image in the retina is extracted by using LBP in effective way. Basically, this system supports very powerful tools when comes to image processing. By using classifier technique the image improves the accuracy level. This is mainly used in the applications of high security. This is a unique system which improves the accuracy in effective way.

\section{References}

[1] M Anna Latha, N Christy Evangeline, S. Sankara Narayanan, 2018 Fourth International Conference on Biosignals, Images and Instrumentation (ICBSII), 2018 Conference Paper, Published on IEEE.

[2] P. T. Karule, Shilpa Joshi , 2017 International Conference on Intelligent Computing and Control (I2C2), 2017 Conference Paper, Published on IEEE.

[3] Siva Teja Kakileti, Krithika Venkataramani, 2016 IEEE International Conference on Image Processing (ICIP), 2016Conference Paper, Published on IEEE.

[4] VeronikaKurilová , JarmilaPavlovičová , Miloš Oravec, Radoslav Rakár, Igor Marček, 2015 International Conference on Systems, Signals and Image Processing (IWSSIP), 2015 Conference Paper, Published on IEEE.

[5] Sohini Roychowdhury, Dara D. Koozekanani, Keshab K. ParhiIEEE Journal of Biomedical and Health Informatics, 2015 Journal Article, Published on IEEE.

[6] Wan Azani B Wan Mustafa, Haniza Yazid, Sazali Bin Yaacob, Shafriza Nisha Bin Basah, 2014 IEEE REGION 10 SYMPOSIUM, 2014 Conference Paper, Published on IEEE.

[7] Murat Ceylan, Hüseyin Yaçar, 36th International Conference on Telecommunications and Signal Processing (TSP), 2013Conference Paper, Published on IEEE. 
[8] Chien-Cheng Lee, Shih-Che Ku, 2012 International Conference on Information Security and Intelligent Control, 2012 Conference Paper, Published on IEEE.

[9] Erhan Bas , Nastaran Ghadarghadar, Deniz Erdogmus, 2011 IEEE International Symposium on Biomedical Imaging: From Nano to Macro, 2011 Conference Paper, Published on IEEE.

[10] D. Wu, Ming Zhang, Jyh-Charn Liu, W. BaumanIEEE Transactions on Biomedical Engineering2006, Journal Article, Published on IEEE. 